\title{
Modeling and Analysis of Drift Error from Stator of A MSSG with Double Spherical Envelope Surfaces
}

\author{
Chao-Jun Xin*, Yuan-Wen Cai*, Yuan Ren ${ }^{\dagger}$ and Ya-Hong Fan**
}

\begin{abstract}
For the newly developing magnetically suspended sensitive gyroscope (MSSG) with double spherical envelope surfaces, it is necessary to analyze the causes of drift error to improve sensing accuracy. This paper builds the models of disturbing torques generated by stator assembly and process errors based on the geometric construction and running regularity of the MSSG, and further reveals the generation mechanism of the drift error. Then the drift error from a single stator magnetic pole is calculated quantitatively with the established model, and the key factors producing the drift error are further discussed. It is proposed that the main approaches in reducing the drift error from stator are guaranteeing the rotor envelope surface to be an ideal spherical and improving the controlling precision of rotor displacement. The common problems associated in a gyroscope with a spherical rotor can be effectively resolved by the proposed method. This analysis provides a reference for the structure optimization and error compensation of a MSSG.
\end{abstract}

Keywords: Magnetically suspended sensitive gyroscope (MSSG), Drift error, Disturbing torque, Spherical rotor, Stator magnetic pole

\section{Introduction}

Due to a variety of great advantages, such as non-contact support, high-speed operation, long life, low friction, oilfree support and so on, active magnetic bearings (AMBs) have been successfully employed for many space missions as ideal attitude control system actuators with high accuracy [1-3]. Traditionally, AMBs are employed as control moment gyroscopes, moment wheels or reaction wheels in space missions [4-5]. In recent years, researchers have begun to develop inertial sensors based on AMBs, named magnetically suspended sensitive gyroscope (MSSG) [6-7]. MSSG is used to measure attitude angle or angular velocities. Compared to traditional mechanical gyroscopes, MSSG has a potential of high-precesion and high-bandwidth, which has a wide prospect of application in aerospace, aviation, navigation, homing, robots, and automobiles.

Y. Maruyama et al. raised the method of inertial measurement using AMBs [6]. They discussed the principles of two-axis angular velocity measurement by AMBs, and experimentally succeeded in using an industrial AMB motor as a gyroscope. The results indicated that there is an upper limit of the bandwidth in two-axis angular velocity measurement. By increasing the feedback gain, the moment of inertia of the rotor and the angular velocity of the rotor about the principal axis, and by decreasing the moment of inertia of the rotor about the orthogonal axis to the principal one, the frequency region for angular velocity

$\dagger$ Corresponding Author: Dept. of Aerospace Equipment, Equipment Academy, China. (jingninan $@ 126 . c o m)$

* Dept. of Aerospace Equipment, Equipment Academy, China.

** Institute of Control Engineering, Beijing, China.

Received: December 16, 2015; Accepted: April 6, 2016 detection can be expanded. Due to lack of effective error compensation, the amplitude error is as high as $10 \%$ in the angular velocity measurement [7-9]. Jiancheng Fang proposed a magnetically suspended control moment gyroscope, which can work in two modes of actuator and attitude sensor [10]. By a PID controller with cross-axis feedback compensation, the angular velocity couplings between the $x$-axes and $y$ - are decoupled. However, the errors of the rotor are not considered in these studies. The assembly errors and process errors of stator are also neglected. Such an estimation method leads to an inaccurate measurement result.

Unlike mechanical bearing gyroscopes, in a MSSG, the AMB itself is utilized as a rotating gyroscope, and the rotor is in the free state of suspension. Only the forces directly acting on the rotor may produce disturbing torques, and cause gyroscope drift errors. There are only three field forces of gravity, inertia force, magnetic force and resistance of the residual gas in the stator house that directly acting on the rotor in an AMB.

Especially in the MSSG studied in this paper, both the magnetic pole envelope surfaces (MPES) of stator and the rotor are spherical surfaces, so as to realize the dynamic decoupling in inertial measurement, thus the measurement precision can be improved. When the geometric centers of the ideal spherical envelope surfaces are coincident, and the device performance parameters of each channel are identical, the magnetic forces over the entire surface of the rotor will pass through its geometric center as it spinning steadily about its polar axis. On this occasion, there are no disturbing torques produced, and no drift errors generated. However, owing to the material inhomogeneity, machining, 
assembly precision, centrifugal deformation and some other reasons, the actual MPESs of stator and rotor will deviate from the ideal sphere [11]. The deviation makes magnetic forces away from the geometric center of the rotor, and disturbing torques acting on geometric center are produced, then the drift errors [12, 13]. Therefore, it is necessary to build the models of disturbing torque generated by stator assembly and process errors of a MSSG.

In recent years, disturbing torques have been deeply researched in superconducting gyroscopes. Chuan $\mathrm{He}$ preliminary analyzed the disturbing torques produced by the asphericity of rotor in a superconducting gyroscope [14]. Aspherical disturbing torques are lead into two categories, one brings from the high-speed spinning centrifugal distortion, and the other from the rotor manufacturing process error. Nevertheless, the author only considers the disturbing torques produced by suspension coils in $\mathrm{Z}$-axis direction, the other factors producing disturbing torque are not in-depth analyzed, nor the drift errors generated from the disturbing torque. Cui Chunyan analyzed disturbing torques produced by the asphericity of rotor, and deduced the model of drift rate in a superconducting gyroscope more systematically. Influence of different factors on the drift errors are brought out quantitatively [15]. The author mainly focuses on the disturbing torques produced by the asphericity of rotor, but disturbing torques from errors of stator have not been sufficiently studied.

Different from the double half-bowl structure of stator in a superconducting gyroscope, the stator of MSSG in our study consists of 6 magnetic poles of spherical envelop surface. Therefore, the generation mechanism of disturbing torque dues to stator errors is completely different from superconducting gyroscope.

Based on the assumption of mass balance of the spherical rotor, we focus our attention on the mechanism and rules of the disturbing torque due to errors of stator in a MSSG. According to the physical mechanism, theoretical model of drift angular velocity from assembly errors and process errors are established, and solutions of reducing drift error are pointed out. This paper is organized as follows. The descriptions of an double spherical enveloped surface MSSG based on AMB is presented, and the drift rate model of spherical rotor is deduced in detail in Section 2. The model of disturbing torque from three assembly errors and two process errors are found in Section 3 and 4, respectively. By substituting the MSSG parameters into the formula, the numerical results are provided in Section 5. Finally, we draw the conclusions in Section 6.

\section{Description of the MSSG and Its Disturbing Torques}

\subsection{Description of the MSSG system}

The MSSG studied in this paper consists of rotor, stator and a stator house which the stator is fixed to. Fig. 1 shows

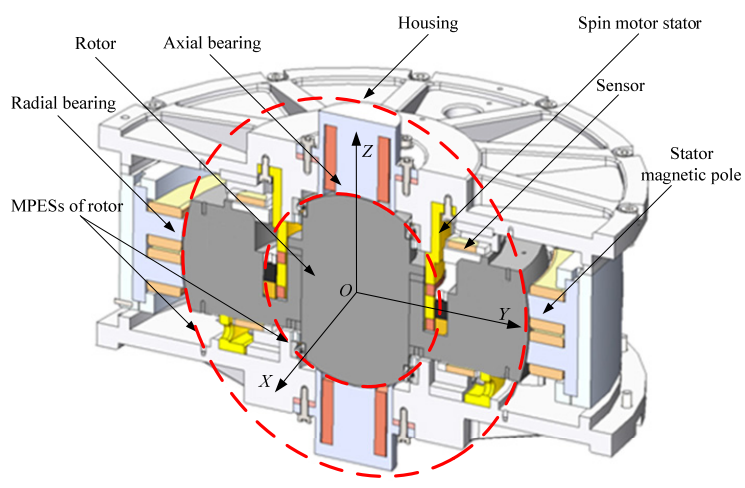

Fig. 1. Configuration of the MSSG

the configuration of the MSSG, and its schematic diagram is drawn in a cross-sectional situation of two dimensions in Fig. 2.

The rotor of the MSSG is a type of spherical in structure. Radiuses of rotor MPESs in axial and radial are different, while the radius is $r_{1}$ in radial and $r_{2}$ in axial. And the rotor is made of magnetic material in whole. There are three pairs of stator magnetic poles in the MSSG. Radius of MPES composed by two pairs of radial stator magnetic poles is $R_{1}$, and that of MPES composed by the pair of axial stator magnetic poles is $R_{2}$. The two envelope surfaces of stator magnetic poles constitute a spherical stator cavity, whose geometric center is coincident with centers of the two spherical stator MPESs. The rotor is located in the central position of the cavity when the system is running in an equilibrium state. Two pairs of radial stator magnetic poles are symmetrically fixed along the coordinate axis in the $O X Y$-plane. The pair of stator magnetic poles in axial are symmetrically attached to the $Z+$ and $Z$-axis direction. The gap between the MPESs of the stator and rotor is $0.35 \mathrm{~mm}$ in nominal.

The stator magnetic poles are electromagnets, so that the bias current and control current would be provided through the coil on the poles, and corresponding magnetic field would be generated. The rotor is levitated in the center of the stator cavity by magnetic pull caused by the interaction of the electromagnetic stator and the rotor. When the rotor is disturbed, its motion state will change. The eddy-current sensors are used to pick up the displacement signals of the rotor. Change in position of the rotor can be detected by the sensors. And the control system will apply a corresponding control current in different coil according to the sensors' signals, which would transfer the rotor back to its original position. To minimize the leakage magnetic interference, the size of the stator magnetic poles is less than that of rotor magnetic poles.

\subsection{Model of gap between magnetic poles}

Fig. 2 shows the block diagram for the MSSG systems in the OYZ-plane. Aside from all the mechanical parts described above, the MSSG system also consists of 


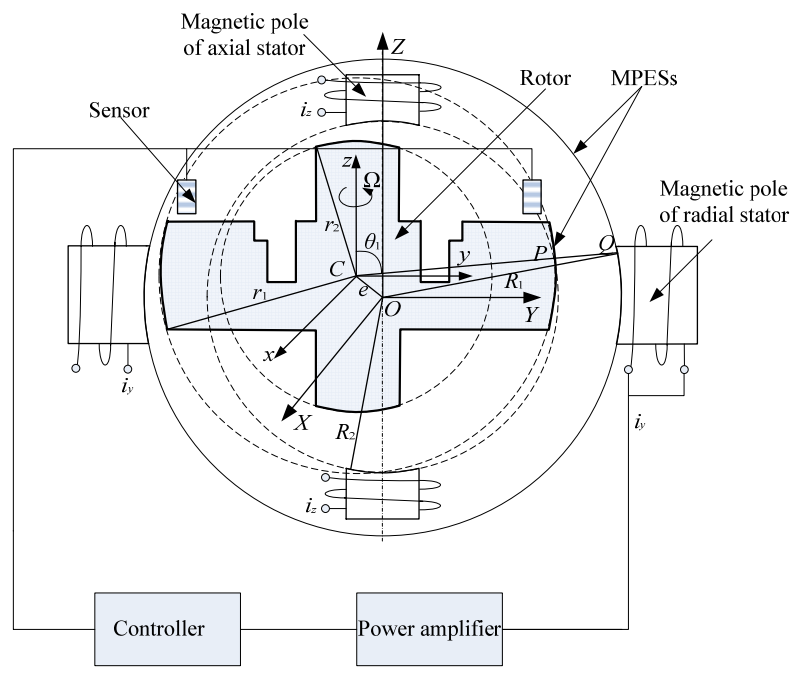

Fig. 2. Block diagram for the MSSG system

controller, power amplifier, signal conditioning circuits, and correlative filters, which are all embedded in the housing.

In Fig. 2, three coordinate systems are introduced. In the rotor coordinate system $(x, y, z), C$ is defined as the geometric center of rotor, and also the origin of the rotor coordinate system. The axes are defined to be aligned with principal axes of inertia of rotor. And $\Omega$ is the spinning speed of the rotor around the major axis of inertia, $z$-axis. Origin of the stator coordinate system $(X, Y, Z)$ is the center of the stator cavity. $A$ spherical coordinate system $\left(R_{1}, \theta, \varphi\right)$ is introduced in correspond with the rectangular coordinate system $(X, Y, Z)$.

The unit vector of spinning axis of rotor $z_{0}$ can be written in the stator coordinate system as

$$
\boldsymbol{z}_{0}=\alpha \boldsymbol{X}_{0}+\beta \boldsymbol{Y}_{0}+\gamma \boldsymbol{Z}_{0}
$$

where $\alpha, \beta, \gamma$ are the direction cosines of $z_{0}$ in the stator coordinate system, and $\boldsymbol{X}_{0}, \boldsymbol{Y}_{0}, \boldsymbol{Z}_{0}$ are the unit vectors of the axes in stator coordinate.

Assuming that point $P$ is on the spherical surface of the rotor, and the line $C P$ intersects the stator magnetic pole surface at point $Q$, the gap size between stator magnetic pole surface and the rotor can be written as

$$
\delta=|P Q|=|C Q|-|C P|
$$

In the case of minute offset, vector $\boldsymbol{C} \boldsymbol{Q}, \boldsymbol{O Q}$ are approximately the same direction, according to the geometric relationship of the vector triangle $C O Q, \delta$ can be calculated as

$$
\delta=R_{1}-r_{1}\left(\theta_{1}\right)-\boldsymbol{e} \cdot \boldsymbol{r}_{0}
$$

in which, $r\left(\theta_{1}\right)$ is the radius vector of point $P$ in rotor coordinate system, and $\theta_{1}$ is the angle between $r$ and $z$-axis. $\boldsymbol{e}$ is the linear displacement vector from $O$ to $C$, named eccentricity, and it can be written as

$$
\boldsymbol{e}=e_{X} \boldsymbol{X}_{0}+e_{Y} \boldsymbol{Y}_{0}+e_{Z} \boldsymbol{Z}_{0}
$$

where $r_{0}$ is the unit vector of $r\left(\theta_{1}\right)$, and it can be described in stator coordinate system $\left(R_{1}, \theta, \varphi\right)$ as

$$
\boldsymbol{r}_{0}=\sin \theta \cos \varphi \boldsymbol{X}_{0}+\sin \theta \sin \varphi \boldsymbol{Y}_{0}+\cos \theta \boldsymbol{Z}_{0}
$$

where $\theta$ is the angle between $O P$ and $Z$-axis, and $\varphi$ is the azimuth of $O P$. Therefore, we can get

$\delta=R_{1}-r_{1}\left(\theta_{1}\right)-e_{X} \sin \theta \cos \varphi-e_{Y} \sin \theta \sin \varphi-e_{Z} \cos \theta$

In order to simplify the analysis, the rotor MPES with radius $r_{1}$ is assumed to be torispherical surface of revolution, and its radius vector in meridian plane can be described by Legendre polynomial series as

$$
r_{1}\left(\theta_{1}\right)=r_{10}+\sum_{n=1}^{\infty} a_{n} P_{n}\left(\cos \theta_{1}\right)
$$

where, $r_{10}$ is the nominal radius of the ideal envelope surface, $a_{n}$ is the aspheric coefficient of each harmonic in describing the envelope surface. $P_{n}\left(\cos \theta_{1}\right)$ is the Legendre polynomial series which is written as

$$
\begin{aligned}
& P_{1}=\cos \theta_{1} \\
& P_{2}=\frac{1}{2}\left(3 \cos ^{2} \theta_{1}-1\right) \\
& P_{n+1}=\frac{1}{n+1}\left[(2 n+1) \cos \theta_{1} P_{n}\left(\cos \theta_{1}\right)-n P_{n-1}\left(\cos \theta_{1}\right)\right] \\
& \quad(n \geq 2)
\end{aligned}
$$

Let $\delta_{0}=R_{10}-r_{10}, R_{10}$ is the nominal radius of the stator MPES with radius $R_{1}$. And substitute (7) into (6), we can get

$$
\begin{aligned}
& \delta=\delta_{0}+\Delta \delta=\delta_{0}-\sum_{n=1}^{\infty} a_{n} P_{n}\left(\cos \theta_{1}\right) \\
& -e_{X} \sin \theta \cos \varphi-e_{Y} \sin \theta \sin \varphi-e_{Z} \cos \theta
\end{aligned}
$$

where

$$
\cos \theta_{1}=\alpha \sin \theta \cos \varphi+\beta \sin \theta \sin \varphi+\gamma \cos \theta
$$

\subsection{Theoretical model of drift error}

The magnetic force element on point $P$ can be expressed as $d F \cdot \boldsymbol{n}_{0}$, where $\boldsymbol{n}_{0}$ is the unit vector of normal line at point $P$. And the radius vector of $C P$ can be written as $r_{1} \cdot \boldsymbol{r}_{0}$. When the rotor MPES is an ideal sphere, $\boldsymbol{r}_{0}$ is coincided with $\boldsymbol{n}_{0}$, that is, the magnetic force passes through the geometric center of the rotor, and there is no disturbing 
torque about the geometric center. However, when the rotor MPES deviates from the ideal sphere and become a torispherical close surface, $\boldsymbol{r}_{0}$ and $\boldsymbol{n}_{0}$ are coincided no longer, disturbing torque is generated by the magnetic force about the geometric center of the rotor, which can be written in differential term as

$$
d \boldsymbol{T}=\boldsymbol{r} \times d \boldsymbol{F}=\left(r_{1} \cdot \boldsymbol{r}_{0}\right) \times\left(d F \cdot \boldsymbol{n}_{0}\right)
$$

So, the disturbing torque equation can be written as [15][16],

$$
\boldsymbol{T}=\iint_{S} \frac{\mu_{0} N^{2} I^{2}}{8 \delta_{0}^{2}}\left(1-\frac{2 \Delta \delta}{\delta_{0}}\right) \cdot\left(\frac{d r_{1}}{d \theta} \cdot \frac{1}{\sin \theta}\right) \cdot \boldsymbol{z}_{0} \times \boldsymbol{r}_{0} d S
$$

where $\mu_{0}$ is the magnetic permeability of vacuum, $N$ is the turns of coil on the stator magnetic pole, $I$ is the current in the coil, $S$ is the area of stator magnetic pole which applied magnetic force on the rotor.

Substituting $(1,5,7,9)$ into (12), converting $d S$ to the spherical coordinate system, and calculating the disturbing torques generated from six stator magnetic poles respectively, the final equation of the disturbing torques is as follow

$$
\boldsymbol{T}=\sum_{i=1}^{6} \iint_{S_{i}}(A \cdot B \cdot C) d \theta d \varphi
$$

where $A=\frac{\mu_{0} N^{2} I^{2} R_{i}^{2}}{8 \delta_{i 0}^{2}}\left(1-\frac{2 \Delta \delta_{i}}{\delta_{i 0}}\right), B=\sum_{n=1}^{\infty} a_{n} P_{n}^{\prime}\left(\cos \theta_{1}\right)$, $C=\left[\begin{array}{c}\beta \cos \theta-\gamma \sin \theta \sin \varphi \\ \gamma \sin \theta \cos \varphi-\alpha \cos \theta \\ \alpha \sin \theta \sin \varphi-\beta \sin \theta \cos \varphi\end{array}\right]$, and $i$ denote the $i$ th magnetic pole of the stator, $R_{i}$ is the nominal radius of the $i$ th stator magnetic pole, $\delta_{i 0}$ is the nominal gap size between the $i$ th stator magnetic pole and the rotor, and $S_{i}$ is the area of the $i$ th stator magnetic pole.

The directional stability of a gyroscope could provide credible azimuth reference to users such as satellites and submarines. However, the accuracy value of the azimuth reference depends largely on the magnitude of the gyro drift errors. The gyro drift errors would result in the azimuth reference deviating from its original position over time, giving rise to the azimuth measuring errors. The magnitude of the gyro drift errors is called drift angular velocity. And the drift angular velocity is the leading indicators to weigh a gyroscope's performance, for it decides the azimuth reference accuracy provided by the gyroscope.

Under the action of the disturbing torque, the spinning axis of rotor will gradually deviated from its original position, resulting drift angular velocity. According to angular momentum law, the drift angular velocity can be expressed as

$$
\left[\begin{array}{c}
\omega_{X} \\
\omega_{Y} \\
\omega_{Z}
\end{array}\right]=\frac{1}{H}\left[\begin{array}{ccc}
0 & \gamma & -\beta \\
-\gamma & 0 & \alpha \\
\beta & -\alpha & 0
\end{array}\right]^{-1}\left[\begin{array}{c}
T_{X} \\
T_{Y} \\
T_{Z}
\end{array}\right]
$$

where the angular momentum can be described with the polar moment of inertia of the rotor $J$ as,

$$
H=J \cdot \Omega
$$

\section{Torques from Assembly Errors of Stator}

\subsection{Linear mismatch errors of magnetic poles}

The linear mismatch errors refer to the misalignment in the axis direction of coordinate system $(X, Y, Z)$ when mounting the stator magnetic poles. The errors changed the relative position of the rotor to the stator magnetic poles, causing changes in the gap $\delta$ and the magnetic field distribution, and ultimately reflected in the generation of disturbing torque.

As is shown in Fig. 3, the linear displacement of stator magnetic pole in $Y+$ direction can be written as

$$
\boldsymbol{\varepsilon}=\varepsilon_{X} \boldsymbol{X}_{0}+\varepsilon_{Y} \boldsymbol{Y}_{0}+\varepsilon_{Z} \boldsymbol{Z}_{0}
$$

In the situation of linear mismatch errors, rules the gap $\delta$ changing is in correspond to subtracting the linear displacement $\varepsilon$ from the original eccentricity e. According to (9), the gap size between the $Y+$ stator MPES and the rotor becomes

$$
\begin{aligned}
& \delta^{\prime}=\delta_{0}-\sum_{n=1}^{n} a_{n} P_{n}\left(\cos \theta_{1}\right)-\left(e_{X}-\varepsilon_{X}\right) \sin \theta \cos \varphi \\
& -\left(e_{Y}-\varepsilon_{Y}\right) \sin \theta \sin \varphi-\left(e_{Z}-\varepsilon_{Z}\right) \cos \theta
\end{aligned}
$$

Substituting (17) into (12), the disturbing torque from the linear mismatch errors can be calculated as

$$
\boldsymbol{T}_{l m_{-} Y+}=\frac{\mu_{0} N^{2} I^{2} R_{1}^{2}}{4 \delta_{0}^{3}} \iint_{S_{X+}}(A \cdot B \cdot C) d \theta d \varphi
$$

where $A=\sum_{n=1}^{\infty} a_{n} P_{n}^{\prime}\left(\cos \theta_{1}\right)$,

$B=\left(\varepsilon_{x} \sin \theta \cos \varphi+\varepsilon_{y} \sin \theta \sin \varphi+\varepsilon_{z} \cos \theta\right)$,

$C=\left[\begin{array}{c}\beta \cos \theta-\gamma \sin \theta \sin \varphi \\ \gamma \sin \theta \cos \varphi-\alpha \cos \theta \\ \alpha \sin \theta \sin \varphi-\beta \sin \theta \cos \varphi\end{array}\right]$.

Disturbing torque in other directions can be calculated in the same way, and the total disturbing torque generated from the linear mismatch errors can be obtained using (13). 


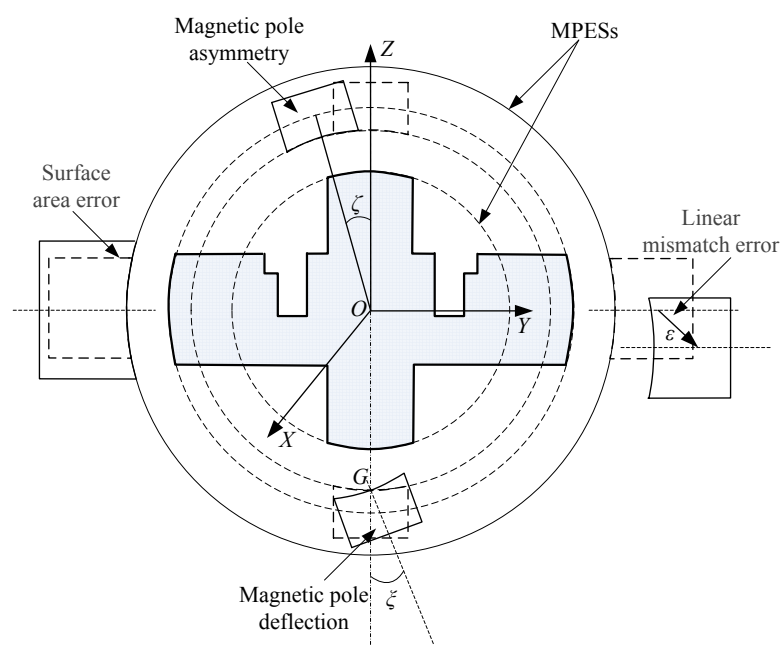

Fig. 3. Stator errors of the MSSG

\subsection{Asymmetry errors of magnetic poles}

The pair of stator magnetic poles which should be installed symmetrically does not achieve complete symmetrical installation, but slide a tiny angle from the ideal location in the envelope surface, which leads to the magnetic pole asymmetry errors. Owing to changes in magnetic pole location, the relative position of the rotor to the stator magnetic poles is altered, so do the gap $\delta$ and the magnetic field distribution, resulting in disturbing torques.

In Fig.3, the magnetic pole in the $Z+$ direction moved a deviation angle of $\zeta$ about the $X$-axis along the envelope surface, resulting in the asymmetry errors with the stator magnetic pole installed in $Z$ - direction.

In magnetic pole asymmetry errors, rule of the gap $\delta$ changing is similar to that of eccentricity $\mathbf{e}$ rotating $\zeta$ about the $X$-axis. And the new eccentricity can be expressed as

$$
\boldsymbol{e}_{1}=\left[\begin{array}{c}
e_{X} \\
e_{Y} \cos \zeta+e_{Z} \sin \zeta \\
-e_{Y} \sin \zeta+e_{Z} \cos \zeta
\end{array}\right]
$$

Thus, gap size between the stator magnetic pole and the rotor can be written as

$$
\begin{gathered}
\delta=-\sum_{n=1}^{n} a_{n} P_{n}\left(\cos \theta_{1}\right)-e_{X} \sin \theta \cos \varphi \quad \begin{array}{r}
\text { Disturbing torque generated from the two } \\
\text { magnetic poles in radial can be calculated sime } \\
\text { the above method. }
\end{array} \\
\boldsymbol{T}_{a_{-} Z+}=\frac{\mu_{0} N^{2} I^{2} R_{2}^{2}}{4 \delta_{0}^{3}} \int_{S_{Z+}}\left(\begin{array}{l}
{\left[\begin{array}{l}
\left.e_{X}\left(\cos \Delta \theta_{Y}-1\right)-e_{Z} \sin \Delta \theta_{Y}\right] \sin \theta \cos \varphi \\
+\left[e_{X} \sin \Delta \theta_{X} \sin \Delta \theta_{Y}+e_{Y}\left(\cos \Delta \theta_{X}-1\right)+e_{Z} \sin \Delta \theta_{X} \cos \Delta \theta_{Y}\right] \sin \theta \sin \varphi \\
+\left[e_{X} \cos \Delta \theta_{X} \sin \Delta \theta_{Y}-e_{Y} \sin \Delta \theta_{X}+e_{Z}\left(\cos \Delta \theta_{X} \cos \Delta \theta_{Y}-1\right)\right] \cos \theta
\end{array}\right)} \\
\times \sum_{n=1}^{\infty} a_{n} P_{n}^{\prime}\left(\cos \theta_{1}\right) \cdot\left[\begin{array}{c}
\beta \cos \theta-\gamma \sin \theta \sin \varphi \\
\gamma \sin \theta \cos \varphi-\alpha \cos \theta \\
\alpha \sin \theta \sin \varphi-\beta \sin \theta \cos \varphi
\end{array}\right] d \theta d \varphi
\end{array}\right.
\end{gathered}
$$
errors in $Z+$ direction as

$$
\text { where } A=\left(\begin{array}{l}
{\left[e_{Y}(\cos \zeta-1)+e_{Z} \sin \zeta\right] \sin \theta \sin \varphi} \\
+\left[e_{Z}(\cos \zeta-1)-e_{Y} \sin \zeta\right] \cos \theta
\end{array}\right),
$$
parts when integrating,

$$
\begin{aligned}
& S_{Z+1}: \quad \theta \in\left(0, \frac{\theta_{0}}{2}+\zeta\right), \quad \varphi \in\left(\begin{array}{ll}
0, & \pi
\end{array}\right) \\
& S_{Z+2}: \quad \theta \in\left(0, \frac{\theta_{0}}{2}-\zeta\right), \quad \varphi \in\left(\begin{array}{ll}
0, & \pi
\end{array}\right)
\end{aligned}
$$

in which $\theta_{0}$ is the circle angle of stator in OYZ-plane. errors can be written as (24).

Substituting (20) into (12), we can get the disturbing torque generated from stator magnetic pole asymmetry

$$
B=\sum_{n=1}^{\infty} a_{n} P_{n}^{\prime}\left(\cos \theta_{1}\right), \quad C=\left[\begin{array}{c}
\beta \cos \theta-\gamma \sin \theta \sin \varphi \\
\gamma \sin \theta \cos \varphi-\alpha \cos \theta \\
\alpha \sin \theta \sin \varphi-\beta \sin \theta \cos \varphi
\end{array}\right] .
$$

In (21), the integral area needs to be divided into two

In general case, asymmetry errors can occur along the envelope surface in any direction. With $\Delta \theta_{X}, \Delta \theta_{Y}$ represent the deviation angles of an axial magnetic pole about the $X$ and $Y$-axis respectively, the eccentricity is converted to be

$$
\boldsymbol{e}_{2}=\left[\begin{array}{c}
e_{X} \cos \Delta \theta_{Y}-e_{Z} \sin \Delta \theta_{Y} \\
e_{X} \sin \Delta \theta_{X} \sin \Delta \theta_{Y}+e_{Y} \cos \Delta \theta_{X}+e_{Z} \sin \Delta \theta_{X} \cos \Delta \theta_{Y} \\
e_{X} \cos \Delta \theta_{X} \sin \Delta \theta_{Y}-e_{Y} \sin \Delta \theta_{X}+e_{Z} \cos \Delta \theta_{X} \cos \Delta \theta_{Y}
\end{array}\right]
$$

Substituting (23) into (12), the disturbing torque generated from axial stator magnetic pole asymmetry

Disturbing torque generated from the two pairs of stator magnetic poles in radial can be calculated similarly using 


\subsection{Deflection errors of magnetic poles}

When the stator magnetic pole is deflected about its own vertex on envelope surface, the disturbing torque will be generated due to the change of magnetic field distribution between the magnetic pole and the rotor.

In Fig. 3, the magnetic pole in the $Z$-direction is deflected about its own vertex $G$ by $\xi$ counterclockwise in $X$-axis. It is equivalent to that the eccentricity e rotates about $G$ by $\xi$ counterclockwise in $X$-axis for the gap $\delta$. The eccentricity after rotation becomes

$$
\boldsymbol{e}_{3}=\left[\begin{array}{c}
e_{X} \\
e_{Y} \cos \xi+\left(e_{Z}+R_{1}\right) \sin \xi \\
-e_{Y} \sin \xi+\left(e_{Z}+R_{1}\right) \cos \xi-R_{1}
\end{array}\right]
$$

Substituting (25) into (12), we can get the disturbing torque generated from magnetic pole deflection errors

$$
\begin{array}{r}
\left(\left[e_{Y}(\cos \xi-1)+e_{Z} \sin \xi\right] \sin \theta \sin \varphi+\left[\left(e_{Z}+R_{1}\right)(\cos \xi-1)-e_{Y} \sin \xi\right] \cos \theta\right) \\
\boldsymbol{T}_{d_{-} Z-}=\frac{\mu_{0} N^{2} I^{2} R_{2}^{2}}{4 \delta_{0}^{3}} \iint_{S_{Z+}} \times \sum_{n=1}^{\infty} a_{n} P_{n}^{\prime}\left(\cos \theta_{1}\right) \cdot\left[\begin{array}{c}
\beta \cos \theta-\gamma \sin \theta \sin \varphi \\
\gamma \sin \theta \cos \varphi-\alpha \cos \theta \\
\alpha \sin \theta \sin \varphi-\beta \sin \theta \cos \varphi
\end{array}\right] d \theta d \varphi
\end{array}
$$

Substituting (25) into (12), we can get the disturbing torque generated from magnetic pole deflection errors Generally, the stator magnetic pole can be deflected about its surface vertex in any direction. For the MSSG in this study, because the stator MPES in axial are symmetrical surfaces of revolution, deflections in Z-axis of which would not produce disturbing torques. But the deflections of other four radial magnetic poles in any direction will produce disturbing torques. Let $\Delta \lambda_{X}, \Delta \lambda_{Y}, \Delta \lambda_{Z}$ represent deflected angles of an magnetic pole about its own vertex in $X$-axis, $Y$-axis, and $Z$-axis respectively. For axial stator magnetic poles, the eccentricity after deflection can be written as (27), where $k$ is the coefficient of magnetic pole assembly, for the magnetic pole in axis positive direction, $k=1$, and for the negative direction $k=-1$. Substituting (27) into (12), the disturbing torque generated from axial stator magnetic poles can be obtained as (28). For the radial stator magnetic poles in $X$ and $Y$-axis, the disturbing torques from deflection can be calculated as (29) and (30).

$$
\begin{aligned}
& \boldsymbol{e}_{4}=\left[\begin{array}{c}
e_{X} \cos \Delta \lambda_{Y}-\left(e_{Z}-k R_{2}\right) \sin \Delta \lambda_{Y} \\
e_{Y} \cos \Delta \lambda_{X}+\left[e_{X} \sin \Delta \lambda_{Y}+\left(e_{Z}-k R_{2}\right) \cos \Delta \lambda_{Y}\right] \sin \Delta \lambda_{X} \\
-e_{Y} \sin \Delta \lambda_{X}+\left[e_{X} \sin \Delta \lambda_{Y}+\left(e_{Z}-k R_{2}\right) \cos \Delta \lambda_{Y}\right] \cos \Delta \lambda_{X}+k R_{2}
\end{array}\right] \\
& \begin{aligned}
& \boldsymbol{T}_{d_{-} Z}=\frac{\mu_{0} N^{2} I^{2} R_{2}^{2}}{4 \delta_{0}^{3}} \int_{S_{Z}}\left(\begin{array}{l}
{\left[e_{X}\left(\cos \Delta \lambda_{Y}-1\right)-\left(e_{Z}-k R_{2}\right) \sin \Delta \lambda_{Y}\right] \sin \theta \cos \varphi} \\
+\left\{e_{Y}\left(\cos \Delta \lambda_{X}-1\right)+\left[e_{X} \sin \Delta \lambda_{Y}+\left(e_{Z}-k R_{2}\right) \cos \Delta \lambda_{Y}\right] \sin \Delta \lambda_{X}\right\} \sin \theta \sin \varphi \\
+\left\{\left[e_{X} \sin \Delta \lambda_{Y}+\left(e_{Z}-k R_{2}\right) \cos \Delta \lambda_{Y}\right] \cos \Delta \lambda_{X}-e_{Y} \sin \Delta \lambda_{X}-e_{Z}+k R_{2}\right\} \cos \theta
\end{array}\right) \\
& \times \sum_{n=1}^{\infty} a_{n} P_{n}^{\prime}\left(\cos \theta_{1}\right) \cdot\left[\begin{array}{c}
\beta \cos \theta-\gamma \sin \theta \sin \varphi \\
\gamma \sin \theta \cos \varphi-\alpha \cos \theta \\
\alpha \sin \theta \sin \varphi-\beta \sin \theta \cos \varphi
\end{array}\right] d \theta d \varphi
\end{aligned} \\
& \boldsymbol{T}_{d_{-} X}=\frac{\mu_{0} N^{2} I^{2} R_{1}^{2}}{4 \delta_{0}^{3}} \int_{S_{X}}\left(\begin{array}{l}
\left\{\left[\left(e_{X}-k R_{1}\right) \cos \Delta \lambda_{Y}-e_{Z} \sin \Delta \lambda_{Y}\right] \cos \Delta \lambda_{Z}+e_{Y} \sin \Delta \lambda_{Z}+k R_{1}-e_{X}\right\} \sin \theta \cos \varphi \\
+\left\{\begin{array}{l}
{\left[\left(k R_{1}-e_{X}\right) \cos \Delta \lambda_{Y} \sin \Delta \lambda_{Z}+e_{Z} \sin \Delta \lambda_{Y} \sin \Delta \lambda_{Z}+e_{Y} \cos \Delta \lambda_{Z}\right] \cos \Delta \lambda_{X}} \\
+\left[\left(e_{X}-k R_{1}\right) \sin \Delta \lambda_{Y}+e_{Z} \cos \Delta \lambda_{Y}\right] \sin \Delta \lambda_{X}-e_{Y}
\end{array}\right\} \sin \theta \sin \varphi \\
+\left\{\begin{array}{l}
{\left[\left(e_{X}-k R_{1}\right) \cos \Delta \lambda_{Y} \sin \Delta \lambda_{Z}-e_{Z} \sin \Delta \lambda_{Y} \sin \Delta \lambda_{Z}-e_{Y} \cos \Delta \lambda_{Z}\right] \sin \Delta \lambda_{X}} \\
+\left[\left(e_{X}-k R_{1}\right) \sin \Delta \lambda_{Y}+e_{Z} \cos \Delta \lambda_{Y}\right] \cos \Delta \lambda_{X}-e_{Z}
\end{array}\right\} \cos \theta
\end{array}\right) \\
& \times \sum_{n=1}^{\infty} a_{n} P_{n}^{\prime}\left(\cos \theta_{1}\right) \cdot\left[\begin{array}{c}
\beta \cos \theta-\gamma \sin \theta \sin \varphi \\
\gamma \sin \theta \cos \varphi-\alpha \cos \theta \\
\alpha \sin \theta \sin \varphi-\beta \sin \theta \cos \varphi
\end{array}\right] d \theta d \varphi
\end{aligned}
$$




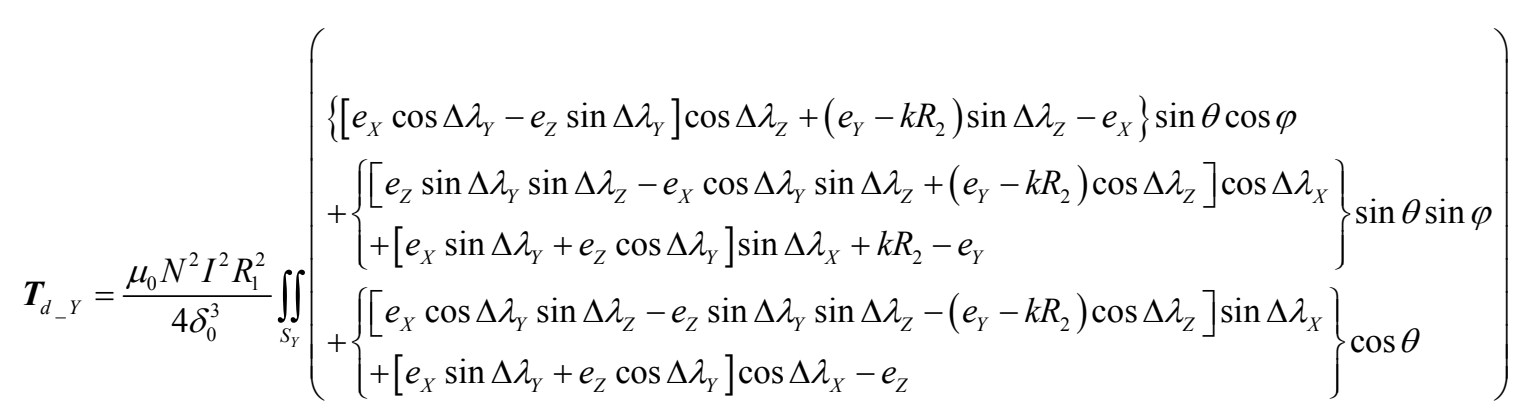

$$
\begin{aligned}
& \times \sum_{n=1}^{\infty} a_{n} P_{n}^{\prime}\left(\cos \theta_{1}\right) \cdot\left[\begin{array}{c}
\beta \cos \theta-\gamma \sin \theta \sin \varphi \\
\gamma \sin \theta \cos \varphi-\alpha \cos \theta \\
\alpha \sin \theta \sin \varphi-\beta \sin \theta \cos \varphi
\end{array}\right] d \theta d \varphi
\end{aligned}
$$

\section{Torques From Process Errors of Stator}

\subsection{Aspherical error of magnetic poles}

Due to processing, fabrication and other reasons, the stator MPES would also become aspherical. Similar to the description of rotor asphericity, radius vector of the stator envelope surface in meridian plane can be described by Legendre polynomial series as

$$
\begin{aligned}
& R_{1}(\theta)=R_{10}+\sum_{n=1}^{\infty} A_{n} P_{n}(\cos \theta) \\
& R_{2}(\theta)=R_{20}+\sum_{n=1}^{\infty} B_{n} P_{n}(\cos \theta)
\end{aligned}
$$

in which $R_{1}(\theta), R_{2}(\theta)$ denote the radiuses vector of the stator MPESs in radial and axial respectively, and $R_{10}$ and $R_{20}$ are the nominal radiuses of the ideal stator MPESs, $A_{n}$, $B_{n}$ are the aspheric coefficient of each harmonic in describing the different envelope surfaces. At this time, the gap $\delta$ can be expressed as

Substituting (32) into (12), we can get the disturbing torque generated from a stator magnetic pole asphericity errors in radial and axial respectively as

$\left.\boldsymbol{T}_{a s_{-} a}=\frac{\mu_{0} N^{2} I^{2} R_{2}^{2}}{4 \delta_{0}^{3}} \iint_{S_{Z} \times}^{\sum_{n=1}^{\infty} A_{n} P_{n}(\cos \theta) \cdot \sum_{n=1}^{\infty} a_{n} P_{n}^{\prime}\left(\cos \theta_{1}\right)} \begin{array}{c}\beta \cos \theta-\gamma \sin \theta \sin \varphi \\ \gamma \sin \theta \cos \varphi-\alpha \cos \theta \\ \alpha \sin \theta \sin \varphi-\beta \sin \theta \cos \varphi\end{array}\right] d \theta d \varphi$

$\boldsymbol{T}_{a s_{-} r}=\frac{\mu_{0} N^{2} I^{2} R_{1}^{2}}{4 \delta_{0}^{3}} \iint_{S_{Z+} \times}^{\sum_{n=1}^{\infty} B_{n} P_{n}(\cos \theta) \cdot \sum_{n=1}^{\infty} a_{n} P_{n}^{\prime}\left(\cos \theta_{1}\right)}\left[\begin{array}{c}\beta \cos \theta-\gamma \sin \theta \sin \varphi \\ \gamma \sin \theta \cos \varphi-\alpha \cos \theta \\ \alpha \sin \theta \sin \varphi-\beta \sin \theta \cos \varphi\end{array}\right] d \theta d \varphi$

\subsection{Area errors of magnetic poles}

Because of processing errors of magnetic pole surfaces, the area of the stator magnetic pole that applied force to rotor would deviate from the desired value. The area error itself does not affect the gap $\delta$, but it will affect the distribution of the magnetic field. On the assumption that there is no magnetic flux leakage and the magnetic field in the gap $\delta$ is distributing uniformly, the area error will directly affect the size of $S$ in (12), and result in disturbing torque.

For the axial stator magnetic poles, the main source of area errors is the error of circle angle $\Delta \theta$, the disturbing torque from which can be calculated as

$$
\boldsymbol{T}_{\Delta S_{-} a}=\int_{0}^{2 \pi} \int_{\theta_{0}}^{\theta_{0}+\Delta \theta}\left[\begin{array}{l}
\frac{\mu_{0} N^{2} I^{2} R_{2}^{2}}{8 \delta_{0}^{2}} \cdot\left(1-\frac{2 \Delta \delta}{\delta_{0}}\right) \cdot \sum_{n=1}^{n} a_{n} P_{n}^{\prime}\left(\cos \theta_{1}\right) \\
\beta \cos \theta-\gamma \sin \theta \sin \varphi \\
\gamma \sin \theta \cos \varphi-\alpha \cos \theta \\
\alpha \sin \theta \sin \varphi-\beta \sin \theta \cos \varphi
\end{array}\right] d \theta d \varphi
$$

For the radial stator magnetic poles, both circle angle error $\Delta \theta$ and azimuth error $\Delta \varphi$ will bring in the area error. Disturbing torques from area error can be expressed as the difference of torques produced by actual area and the ideal area of the magnetic pole surfaces.

$$
\boldsymbol{T}_{\Delta S_{-} r}=\frac{\mu_{0} N^{2} I^{2} R_{1}^{2}}{8 \delta_{0}^{2}}\left(\int_{\varphi_{0}}^{\varphi_{1}+\Delta \varphi \theta_{1}+\Delta \theta} \int_{\theta_{0}}^{\varphi_{1}} d \boldsymbol{T}-\int_{\varphi_{0}}^{\theta_{\theta_{0}}} \int_{\theta_{0}} d \boldsymbol{T}\right)
$$

\section{Results and Analysis}

To simplify operations, the MSSG is assumed to working on a stabilized platform, so that $\alpha \approx 0, \beta \approx 0, \gamma \approx 1$. On the assumption that there is no magnetic flux leakage, errors of a single stator magnetic pole does not affect other poles. Take the $Y+$ stator magnetic pole of the MSSG 
studied in this paper for example, the integral area of the magnetic pole are

$\varphi \in\left(\frac{\pi}{2}-0.5367, \frac{\pi}{2}+0.5367\right), \theta \in\left(\frac{\pi}{2}-0.063, \frac{\pi}{2}+0.063\right)$

Ignore the third-order trace and above, disturbing torques from different errors of stator are as follows,

$$
\begin{aligned}
& \boldsymbol{T}_{l_{-} Y+}=\kappa\left[\begin{array}{ccc}
-0.0553 a_{1} & -0.0675 a_{1} & -0.0004 a_{2} \\
0.0675 a_{1} & 0.0553 a_{1} & 0.0004 a_{2} \\
0 & 0 & 0
\end{array}\right]\left[\begin{array}{c}
\varepsilon_{X} \\
\varepsilon_{Y} \\
\varepsilon_{Z}
\end{array}\right] \\
& \boldsymbol{T}_{a \quad Y+}=\kappa \times \\
& {\left[\begin{array}{cc}
0.0553 a_{1} e_{Z}-0.0004 a_{2} e_{X} & -0.0553 a_{1} e_{Y}+0.0675 a_{1} e_{X} \\
-0.0675 a_{1} e_{Z}+0.0004 a_{2} e_{X} & -0.0553 a_{1} e_{X}+0.0675 a_{1} e_{Y} \\
0 & 0
\end{array}\right]\left[\begin{array}{c}
\Delta \theta_{X} \\
\Delta \theta_{Z}
\end{array}\right]}
\end{aligned}
$$

where $\kappa=\frac{\mu_{0} N^{2} I^{2} R_{1}^{2}}{8 \delta_{0}^{3}}$.

In calculating the disturbing torque generated from deflection error, in consideration of the deflection angle is very small, we assumed that $\cos \Delta \lambda_{i} \approx 1,(i=X, Y, Z)$ and $\sin \Delta \lambda_{i} \approx \Delta \lambda_{i},(i=X, Y, Z)$, then the disturbing torque can be written as (39).

Disturbing torque from area errors is very complex. Area error is coupled with all of the error factors, including eccentricity, rotor asphericity, original circle angle, original azimuth, circle angle error $\Delta \theta$ and azimuth error $\Delta \varphi$. And the error factors are also coupled each other. Similar to calculating the disturbing torque from deflection errors, we assumed that $\sin \left(\theta_{1}+\Delta \theta\right) \approx \sin \theta_{1}, \cos \left(\theta_{1}+\Delta \theta\right) \approx \cos \theta_{1}$ $\sin \left(\varphi_{1}+\Delta \varphi\right) \approx \sin \varphi_{1}, \quad \cos \left(\varphi_{1}+\Delta \varphi\right) \approx \cos \varphi_{1}$ and the disturbing torque from area error is given in (41).

$$
\begin{aligned}
& \boldsymbol{T}_{d_{-} Y+}=\kappa\left[\begin{array}{ccc}
-0.0675 a_{1} e_{Z}+0.0004 a_{2} e_{Y} & 0.0553 a_{1} e_{Z}-0.0004 a_{2} e_{X} & 0.0044 a_{1}-0.0553 a_{1} e_{Y} \\
0.0553 a_{1} e_{Z}-0.0004 a_{2} e_{Y} & -0.0675 a_{1} e_{Z}+0.0004 a_{2} e_{X} & -0.0053 a_{1}+0.0675 a_{1} e_{Y} \\
0 & 0 & 0
\end{array}\right]\left[\begin{array}{l}
\Delta \lambda_{X} \\
\Delta \lambda_{Y} \\
\Delta \lambda_{Z}
\end{array}\right] \\
& \boldsymbol{T}_{a S_{-} Y+}=\kappa\left[\begin{array}{cc}
0.0004 a_{2} & -0.0453 a_{1} \\
-0.0004 a_{2} & 0.0453 a_{1} \\
0 & 0
\end{array}\right]\left[\begin{array}{l}
b_{1} \\
b_{2}
\end{array}\right] \\
& \boldsymbol{T}_{S_{-} Y+}=\kappa\left[\begin{array}{ccl}
-0.2684 a_{1} e_{Y} & -0.0629 a_{1} e_{Y} & 0.0014 a_{1}^{2}-0.0021 a_{2}^{2}-0.0530 a_{1} a_{2} \\
-0.2349 a_{1} e_{X} & & -0.0024 a_{1} e_{X}-0.0037 a_{1} e_{Y}-0.0020 a_{1} e_{Z} \\
& & 0.0014 a_{1}^{2}-0.0022 a_{2}^{2}-0.0037 a_{1} e_{X} \\
+0.1695 a_{1} e_{Y} & -0.4498 a_{2} e_{X} & +0.0039 a_{1} e_{Y}+0.0014 a_{1} e_{Z}+0.0088 a_{2} e_{Y} \\
+0.2673 a_{1} e_{X} & +0.5189 a_{1} e_{X} & -0.0002 a_{2} e_{Z} \\
0 & 0 & 0
\end{array}\right]\left[\begin{array}{c}
\Delta \theta \\
0 \\
0
\end{array}\right.
\end{aligned}
$$

From the above formulas of (37) to (41), we can see that the disturbing torques generated from stator magnetic poles would apply to rotor in $X$ - and $Y$-axis, causing drifting of rotor spinning axis, but the disturbing torque in $Z$-axis is zero, thus has no affection on the spinning speed of rotor. The factor of rotor asphericity plays a decisive role in the generation of disturbing torque. When the envelope surfaces of rotor are ideal spherical, the disturbing force produced by the errors of stator will pass through the geometric center of rotor, and no disturbing torques appears. Eccentricity coupled with the aspheric coefficients of rotor is very important in (38), (39) and (41). Therefore, try to reduce the rotor asphericity and eccentricity, while take the rotor axes of inertia in the same direction of stator axes [14], the disturbing torque can be decreased significantly.

Fig. 4 and Fig. 5 shows the relationship of the disturbing torque with rotor aspheric coefficients and the rotor eccentricity. From Fig. 4, it can be found that, the amplitude of the disturbing torque affected by rotor aspheric coefficients is about $10^{-8} \mathrm{~N} \cdot \mathrm{m}$. And as the increasing of $a_{1}$ and $a_{2}$, the value of disturbing torque increases significantly in $X$ - and $Y$-direction, however, the increment of disturbing torque along with $a_{1}$ is far surpass the along with $a_{2}$. When the aspheric coefficients decrease to zero, the disturbing torque will disappear.

According to Fig. 5, we can discover that, the amplitude of the disturbing torque affected by the rotor eccentricity is 2 orders magnitude less than that of aspheric coefficients. The value of disturbing torque is increasing along with ex 

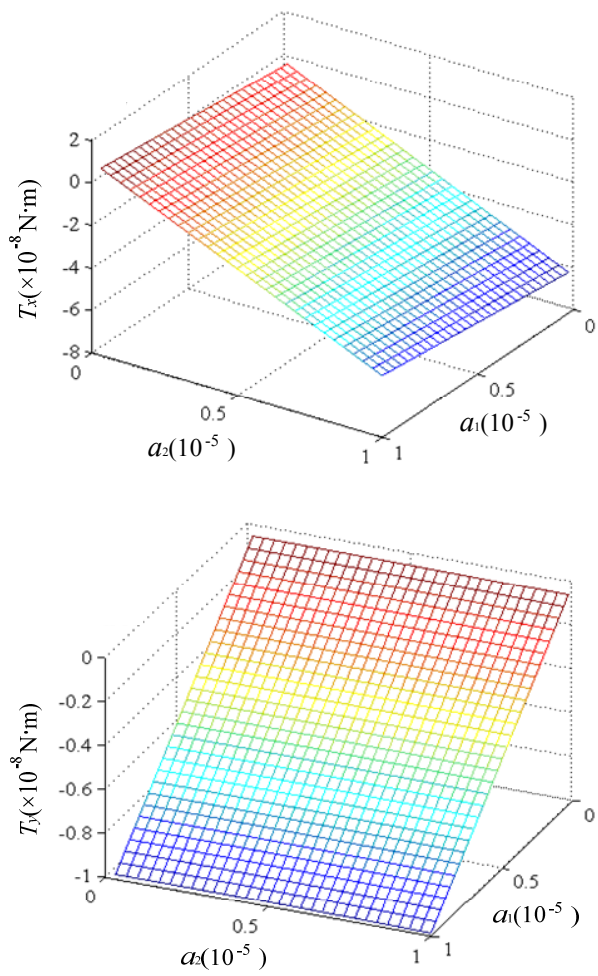

Fig. 4. Relationship of disturbing torque and rotor aspheric coefficients

and $e_{y}$ in $X$ - and $Y+$ direction respectively. And the minimum value can be obtained if both $e_{x}$ and $e_{y}$ are zero.

Among all the errors of stator magnetic poles, errors of linear mismatch and stator asphericity are in correlation with the rotor asphericity, but independent of eccentricity in generating the disturbing torque, as in (37) and (40). However, errors of linear mismatch, asymmetry, deflection, and stator asphericity are all coupled with the aspheric coefficients of rotor, and the main source of the disturbing torque is the coupling with first harmonic, as is shown in (30) and (40).

According to (14), the drift velocity generated from stator magnetic pole errors in $Y+$ direction can be written as (42), in which the error coefficient matrix $\kappa\left[\begin{array}{lllll}\mathbf{A} \boldsymbol{T}_{l_{-} Y+} & \mathbf{A} \boldsymbol{T}_{a_{-} Y+} & \mathbf{A} \boldsymbol{T}_{d_{-} Y+} & \mathbf{A} \boldsymbol{T}_{a s_{-} Y+} & \mathbf{A} \boldsymbol{T}_{S_{-} Y+}\end{array}\right]$ is the combination of each error coefficient matrix in (37) to (41), in the error matrix $\left[\begin{array}{llll}c_{1} & c_{2} & \cdots & c_{13}\end{array}\right]^{\mathrm{T}}, c_{i}$ is defined as (43).

$$
\left[\begin{array}{c}
\omega_{X} \\
\omega_{Y} \\
\omega_{Z}
\end{array}\right]=\frac{\kappa}{H}\left[\mathbf{A} \boldsymbol{T}_{l_{-} Y+} \mathbf{A} \boldsymbol{T}_{a_{-} Y_{+}} \mathbf{A} \boldsymbol{T}_{d_{-} Y+} \mathbf{A} \boldsymbol{T}_{a s_{-} Y+} \mathbf{A} \boldsymbol{T}_{S_{-} Y+}\right]\left[\begin{array}{c}
c_{1} \\
c_{2} \\
\vdots \\
c_{13}
\end{array}\right]
$$

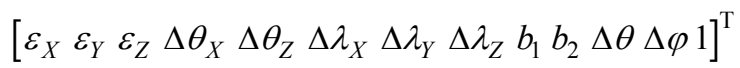

Assume the linear mismatch is $10 \mu \mathrm{m}$, and the angular
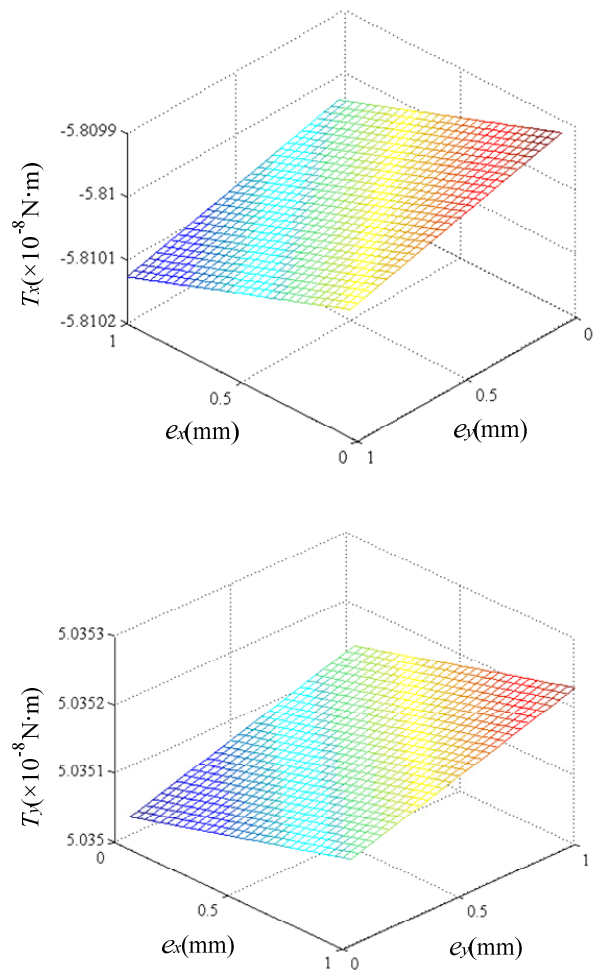

Fig. 5. Relationship of disturbing torque and rotor eccentricity

errors are $0.001^{\circ}, a_{1}=12.392 \times 10^{-6}, a_{2}=14.424 \times 10^{-6}$, $b_{1}=2.404 \times 10^{-6}, b_{2}=4.512 \times 10^{-6}$, the final drift angular velocity can be calculated as

$$
\left[\begin{array}{c}
\omega_{X} \\
\omega_{Y} \\
\omega_{Z}
\end{array}\right]=\left[\begin{array}{c}
-0.8351 \times 10^{-3 \circ} / \mathrm{h} \\
0.7241 \times 10^{-3 \circ} / \mathrm{h} \\
0
\end{array}\right]
$$

Owning to the limitation of space, we only analyzed the drift angular velocity generation mechanism of stator magnetic pole in $Y+$ direction, just as shown in (42), however, the drift angular velocity generated from other stator magnetic poles can be calculated in the same way, and the general drift angular velocity produced by the errors of stator is the sum of drift angular velocities generated from all six stator magnetic poles.

\section{Conclusion}

According to the physical mechanism of a dual spherical envelope surface MSSG, the disturbing torque generated from stator magnetic pole errors is analyzed. Analytical models of the disturbing torque have been founded. According to the analytical models, the disturbing torque and the drift angular velocity of the stator magnetic pole in $Y+$ direction have been discussed particularly. Theoretical 
analysis shows that the error of rotor asphericity is the most critical factor leading to the above mentioned disturbing torques. And it is the fundamental cause of disturbing torque that the error of rotor asphericity is coupled with various errors of the stator. Therefore, it is an important means to reduce the drift angular velocity that guaranteeing the rotor envelope surface to be an ideal spherical when it spinning steadily by increasing the machining precision. The rotor eccentricity coupled with the errors of asymmetry, deflection, and area is another important cause of disturbing torque. The first harmonic of rotor asphericity corresponds to the translation of rigid body axially, which is the main source of disturbing torques from linear mismatch and stator asphericity by coupled with them. Theoretically, drift velocity introduced by factors mentioned above can be significantly decreased by controlling the rotor spinning at the center of the stator cavity.

The research work of this paper achieved the drift angular velocity generation mechanism of stator magnetic pole in a kind of MSSG, and expanded the understanding of the disturbing torques generated from the errors of stator. What we discussed in this paper will provide an available reference for the structure optimization, drift angular velocity measurement and error compensation of a MSSG.

\section{References}

[1] Fang Jiancheng, Zheng Shiqiang and Hang Bang, "Amb Vibration Control for Structural Resonance of Double-Gimbal Control Moment Gyro with HighSpeed Magnetically Suspended Rotor," IEEE/ASME Trans. Mechatronics, vol.18, no.1, pp.32-43, 2013.

[2] Ren Yuan and Fang Jiancheng, "Current-sensing Resistor Design to Include Current Derivative in PWM H-bridge Unipolar Switching Power Amplifiers for Magnetic Bearings," IEEE Trans. Ind. Electron., vol.59, no.12, pp.4590-4600, 2012.

[3] Ren Yuan and Fang Jiancheng, "High-precision and Strong-robustness Control for an MSCMG Based on Modal Separation and Rotation Motion Decoupling Strategy," IEEE Trans. Ind. Electron., vol. 61, no. 12, pp. 1539-1551, 2014.

[4] Ren Yuan and Fang Jiancheng, "Modified Cross Feedback Control for a Magnetically Suspended Flywheel Rotor with Significant Gyroscope Effects," Mathematical Problems in Engineering, vol. 2014, no. 1, pp. 1-11,2014.

[5] Fang Jiancheng, Ren Yuan and Fan Yahong, "Nutation and Precession Stability Criterion of Magnetically Suspended Rigid Rotors with Gyroscopic Effects Based on Positive and Negative Frequency Characteristics," IEEE Trans. Ind. Electron., vol. 61, no. 4, pp. 2003-2014, 2014.

[6] Maruyama Y, Takasaki M, Ishino Y, et al, "Basic
Study on Gyroscopic Sensor Using Active Magnetic Bearing," in Proceedings of 10th International Symposium on Magnetic Bearings, Martigny, Switzerland, Aug. 2006.

[7] Maruyama Y, Mizuno T, Takasaki M, et al, "An Application of Active Magnetic Bearing to Gyroscopic and Inertial Sensors," Journal of System Design and Dynamics, vol.2, no.1, pp.155-164, 2008.

[8] Maruyama Y, Mizuno T, Takasaki M, et al, "Extension of Measurement Bandwidth in an AMBbased Gyroscopic Sensor," Mechatronics, no. 1, pp. 1261-1268, 2009.

[9] Maruyama Y, Mizuno T, Takasaki M, et al, "Proposal of A New Configuration for Magnetically Suspended Gyro," IEEE Trans. Ind. Electron, vol. 61, no. 4, pp. 1911-1916, 2009.

[10] Fang Jiancheng, Zheng Shiqiang and Han Bang, "Attitude Sensing and Dynamic Decoupling Based on Active Magnetic Bearing of MSDGCMG," IEEE Trans. Instrum. Meas., vol. 61, no. 2, pp. 338-348, 2012.

[11] Hu Xinning, Wang Qiuliang, Cui Cunyan, et al, "Torque Compensation System Design for A Spherical Superconducting Rotor," IEEE Trans. Instrum. Meas., vol. 63, no. 12, pp. 2789-2794,2014.

[12] He Chuan, and Wang Qiuliang, "Force Characteristics Analysis on A Superconducting Sphere Suspended by Spherical Coils," Cryogenics, vol. 47, no.7, pp.413417, 2007.

[13] He Chuan, Wang Qiuliang, and Li Xian. "Modeling of the Superconducting Suspension System with Shaping Blocks," IEEE Trans. Appl. Supercond., vol. 20, no. 1, pp. 47-51, 2010.

[14] He Chuang, Wang Qiang, Li Chunsheng., et al, "Analysis of the Torque on a Superconducting Spinning Sphere from Aspheric Factors," IEEE Trans. Appl. Supercond., vol. 17, no. 2, pp. 2174-2177, 2007.

[15] Cui Chunyan, Hu Xinning, Chen Junsheng, et al, "Analysis of Magnetic Disturbance Torque and Drift Error in A Superconducting Suspension System," Acta Phys. Sin., vol. 64, no. 1, pp. 1-11, 2015 (in Chinese).

[16] Fang Jiancheng and Ren Yuan. Magnetically Suspended Control Moment Gyroscope Technology, Beijing, China: National defense industry press, 2014.

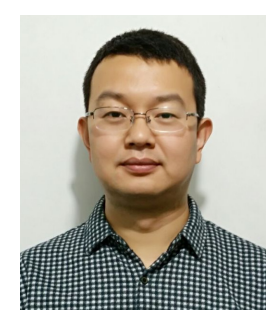

Chao-Jun Xin He received his B.S., M.S. degree in aerospace engineering from Equipment Academy, China, in 2003 and 2008, respectively. His research interests are guide, navigation and control. 


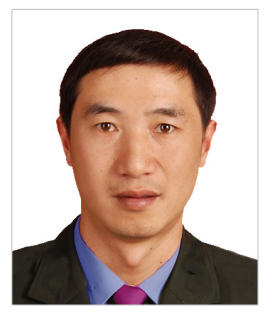

Yuan-Wen Cai He received the B.S. and M.S. degrees in electrical engineering from National University of Defense Technology in 1988 and 1990. And he received the Ph.D. degrees from Beihang University in 2005. He has been a professor in the Department of Aerospace Eqipment, Eqipment Academy. His research interest is aerospace launch and measurement.

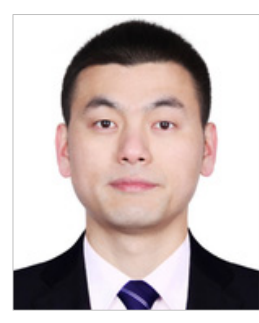

Yuan Ren He received his B.S. degree from Ordnance Engineering College in 2003 and the M.S. degree from Jiangsu University in 2007. He received the Ph.D. degree from Beihang University in 2013. His main research interests are in the areas of advanced inertia measurement and control.

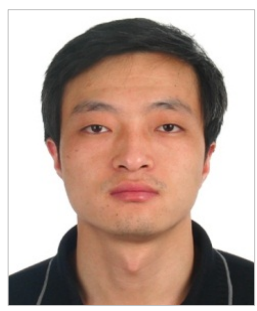

Ya-Hong Fan He received his B.S. degree from Shaanxi University of Technology in 1996. And he received his M.S. and Ph.D. degrees from Beihang University in 2003 and 2011 respectively. His research interests include magnetically suspended control moment gyroscope analysis and design, control and condition monitoring. 